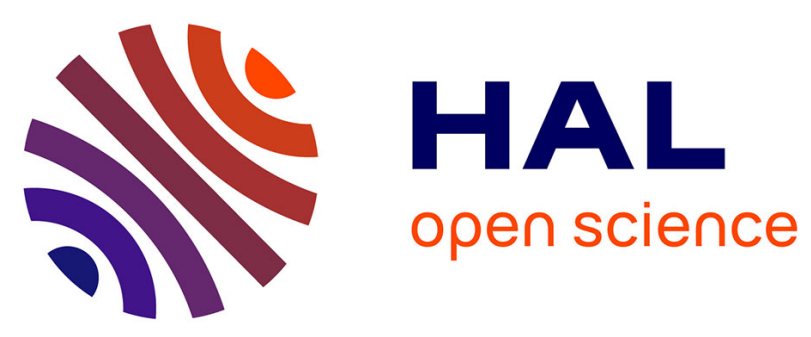

\title{
Les roses des vents latines
}

Philippe Fleury

\section{- To cite this version:}

Philippe Fleury. Les roses des vents latines. Euphrosyne. Revista de filologia clássica, 1991, 19, pp.61-88. hal-01609482

\section{HAL Id: hal-01609482 \\ https://hal.science/hal-01609482}

Submitted on 3 Oct 2017

HAL is a multi-disciplinary open access archive for the deposit and dissemination of scientific research documents, whether they are published or not. The documents may come from teaching and research institutions in France or abroad, or from public or private research centers.
L'archive ouverte pluridisciplinaire HAL, est destinée au dépôt et à la diffusion de documents scientifiques de niveau recherche, publiés ou non, émanant des établissements d'enseignement et de recherche français ou étrangers, des laboratoires publics ou privés. 
INSTITUTO NACIONAL

DE INVESTIGAÇÃO CIENTIFICA

CENTRO DE ESTUDOS CLÁSSICOS

FACULDADE DE LOTA

\section{EVPHROSYNE}

REVISTA DE FILOLOGIA CLÁSSICA

NOVA SÉRIE - VOLUME XIX

SEPARATA

PH. FLEURY

Les roses des vents latines

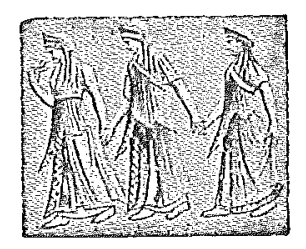

$\operatorname{MCMXCY}$ 


\section{Les roses des vents latines}

Pline l'Ancien écrivait au Livre II de son Histoire Naturelle: «plus de vingt anciens auteurs grecs ont publié des observations 〈sur les vents〉; c'est une chose qui m'étonne fort» ${ }^{1}$. La littérature météorologique antique semble en effet avoir été abondante ${ }^{2}$, de même qu'est relativement abondante la littérature moderne sur les roses des vents grecques et romaines ${ }^{3}$. Que des peuples sans cesse au contact de l'élément marin, pour lesquels le moyen de communication entre les îles, entre la Grèce et l'Italie ou entre l'Asie et l'Europe était la mer, s'intéressent aux vents est en fait une chose aisément explicable ${ }^{4}$. Le sens du vent avait une 1 Puine, Nat. 2, 117.

avant Pline, Vitruve fait également état d'une bibliographie sur le sujet (cf. 1, 6, 4: nonnullis placuit esse uentos quattuor ...).

3 Sur les roses des vents proprement dites: $\mathrm{K}$, von RAUMER, «Die Windrose der Griechen und Römer», RhM, 5, 1837, pp. 497-521; F. A. UKERT, «Uber die Windscheiben und Winde der Griechen und Römer», Zeitschrift für Altertums-Wiss., 8, 1841, n. 15-18; M. D'AvezaC, Aperçus historiques sur la Rose des vents, Rome, 1877; G. KAirbBL, «Antike Windrosen», Hermes, 20, 1885, pp. 579-624; H. STEINMETZ, De uentorum descriptionibus apud Graecos Romanosque, Diss. Göttingen, 1907; A. RRHM, «Griechische Windrosen»,

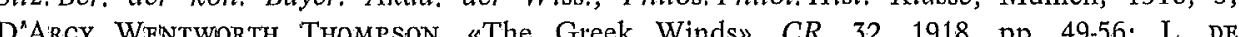
SAUSSURB, "L'origine de la rose des vents et linnvention de la boussole», dans $G$. FEprosp, Introduction à l'astronomie nautique arabe (Bibliothèque des Géographes arabes, t. 1), Paris, P. Geuthner, 1928, pp. 31-127; M. TALLQUIST, «Himmelsgegenden und Winde», Studia Orient. 1, Helsingfors (1928), pp. 105-185; P. D'HérouviLII, «Météorologie agronomique selon Virgile. Les vents», LEC, 1941, pp. 321-328; K. NirLSEN, «Remarques sur les noms grecs et latins des vents et des régions du ciel», C\&M, 7, 1945, pp. 1-113; J.F. MASSELINk, De griek-romeinse Windrose, Utrecht Nijmegen, 1945; parmi les travaux que nous venons de citer, rarticle de $K$. NIELSEN permet de se passer des précédents, mais il n'est pas exempt G. SchмidT dans la Real-Encyclopädie de Pauly et Wissowa (su "Winde»), le commentaire de I. BeAurgu dans son édition du Livre II de Pline 1'Ancien pourr la Collection des Universités de France (Paris, Les Belles Lettres, 1950), sans parler des études plus générales dans le domaine de la météorologie ancienne. Sur les aspects archéologiques des représentations de vents dans 1'Antiquité, voir K. NeusER, Anemoi. Studien zur Darstellung der Winde und

4 Les 
grande importance pour ces navires qui n'avaient pas les capacités de remontee au vent des voiliers modernes. Mais les constructions litte raires des roses des vents $s$ articulent dans une autre problématique: celle de la définition et de l'orientation des régions du ciel. Aujourd'hu deux systèmes coexistent. L'un, le plus courant, utilise les dénominations cardinales d'origine germanique, introduites en françis au $\mathrm{XII}^{\mathrm{e}}$ silcte par l'intermédiaire de l'anglais: est, ouest, sud, nord ${ }^{5}$, et leurs combihaisons à deux niveaux: nordest, nordouest, sudest, sudouest. Mais, dans la navigation maitime et árienne, la divion

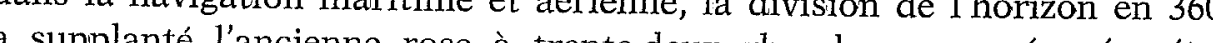
a supp à l'ancin quement à partir des combinaisons à quatre niveaux. nord, nord-es l'ard-nord-est, nord quart de nord-est, nord-est quart de nord, etc. Dans Antiquite la division du ciel s'appuie sur quatre lignes, déterminan au total huit points sur lhorizon: la ligne équinoxiale levant-couchant, a perpendiculaire nord-sud et les deux lignes solsticiales levant d'étécouchant d'ete, levant dhiver-couchant d'hiver. La division purement géométrique n'apparaît que sur les roses des vents. Noms de vents et noms d'orientation sont intimement liés et ces questions sont traitées à linterieur du large cadre de la physique, en liaison avec deux problèmes que nous $\mathrm{n}$ aborderons pas ici: l'origine du vent et les vents locaux ou particuliers.

L'objet du présent travail est d'extraire dans la littérature latine les roses des vents données dans une perspective scientifique ou technique ${ }^{6}$ puis de les présenter de façon uniforme et cohérente en évitant de les attacher systématiquement à la tradition grecque. Nous avons été ainsi amené à remanier les traductions françaises quand elles existaient, quelquefois aussi nous avons rétabli le texte des manuscrits quand les edrecque avalent corige en fonction d'un nom existant sur les roses grecques ou en fonction d'un nom plus habituellement employé. Il nous a paru utile de rassenbler un matériau dispersé chez neuf auteurs différents et de ne négliger aucune des roses décrites: sauf erreur de transmission manuscrite, elles correspondent toutes à un état de la langue à un moment donné, dans un lieu donné. Leur diversité s'explique par des différences locales et chronologiques.

${ }_{5}$ Est (all, Ost, angl, east) < i.e. "ausos; cf. skr. ŭsas, gr. n்ंws, lat, aurora < "ausos-a.

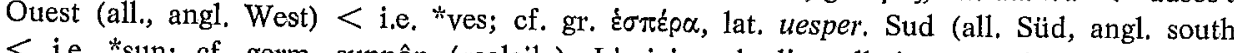
< l.e, "sun; cf. germ. sunnôn («soleil»). L'orighte de lappellation «nord» est contestée:

des poètes notames des vents peuvent aussi être reconstitúés à partir d'autres textes, ceux
Vitruve $(1,6,4-10)$

Vitruve décrit les roses des vents au Livre I du De architectura dans des chapires consacrés à l'urbanisme. L'hygiène est pour lui une préoccupation fondamentale et après avoir conseille de choisir un lieu sain, élevé, exempt de brumes et de gelées, expose à une orientation ni chaude ni froide, mais tempérée, éloigné des marais ${ }^{7}$, il préconise d'orienter le réseau des voies en fonction des vents afin de ne pas les laisser enfiler les ruelles comme c'est le cas, dit-il en exemple, à Mytilène. Au chapi-

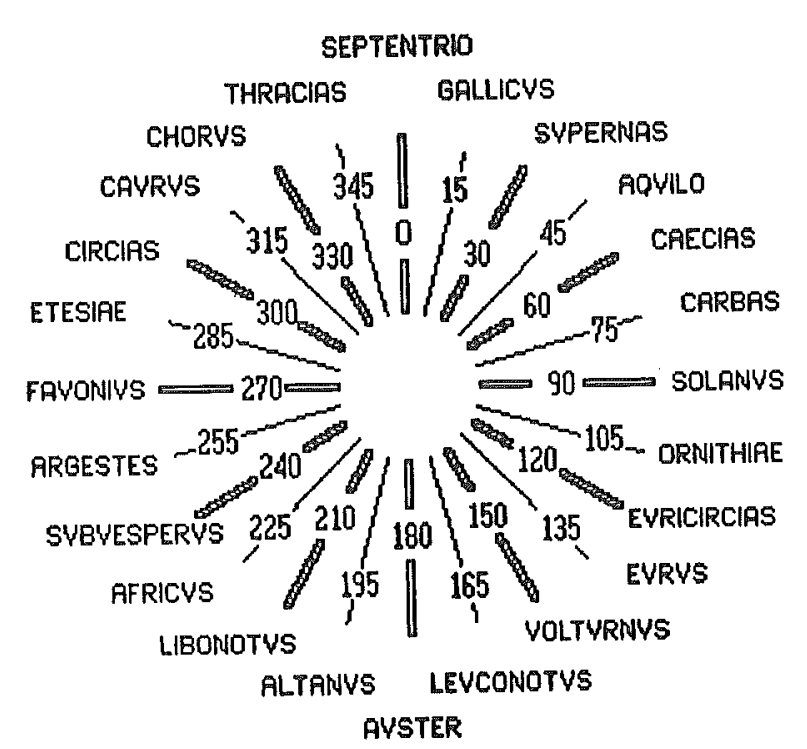

Fig. 1. La rose à vingt-quatre vents de Vitruve (I ${ }^{\text {er }}$ siècle $a . C$.)

tre VI il donne une définition du vent et explique ses effets nocifs quand il est trop abondant, puis il expose successivement trois roses: une rose quatre vents, une rose à huit vents (sur laquelle il fait reposer son

\section{VITr. 1, 4, 1}

8 Nous normalisons les représentations de roses des vents en orientant toujours le nord pour la construction de Sénèque (cf. infra). D'autre part, même quand il est manifeste que vers le haut de la feuille à la manière moderne, mais ce n'était pas systématique chez les Anciens qui devaient fréquemment orienter l'est vers haut; c'est evident par exemple les auteurs ne cherchent pas à combiner la division géométrique du cercle avec la position des points solsticiaux (Aulu-Gelle par exemple), nous adoptons la valeur approchée de $30^{\circ}$ de part et d'autre 
système d'orientation) et une rose à vingt-quatre vents qu'il donne à titre d'excursus:

Selon quelques auteurs il y a quatre vents: le Solanus qui souffle du levant equinoxial, lAuster du midi, le Favonius du couchant equinoxial, le Septentrion du nord. Mais ceux qui firent des recherches plus approfondies enseignèrent qu'il y en avait huit; c'est le cas essentiellement d'Andronicus de Cyrrhos qui en exemple érigea mème a Alhènes une face ̀̀ la direction de leur souffle, des sculptures représentant chaque vent $[\ldots .$.$] C'est pourquoi furent placés entre le Solanus et l'Auster,$ l'Eurus qui souffle du levant d'hiver; entre l'Auster et le Favonius, l'Africus qui souffle du couchant d'hiver; entre le Favonius et le Septentrion, le Caurus que plusieurs nomment Chorus; entre le Septentrion et le Solanus, l'Aquilon [...] Ceux qui connaissent beaucoup de noms de vents s'etonneront peut-être qu'il n'y ait que huit vents clans Cyrène, ques, a trouvé, par la course du soleil, les ombres du gnomon à l'équinoxe et, à partir de l'inclinaison du ciel, que la circonférence de la terre était de deux cent cinquante deux mille stades, c'est-à-dire trente et un million cinq cent mille pas, donc l'octant occupé manifestement par chaque vent est de trois millions neuf cent trente sept mille cinq cents pas; ainsi ils ne devront pas s'étonner si un même vent se déplaçant sur un si vaste espace, avec des changements de direction et des sauts, produit plusieurs variétes par variation de son souffle. C'est Leuconotus et l'altanus, à droite et à gauche de l'Africus, le Libonotus et le Subvesperus; autour du Favonius, l'Argestes et à certaines époques les Étésiens; sur les flancs du Caurus, le Circias et le Chorus; autour du Septentrion, le Thracias et le Gallicus; à droite et à gauche de l'Aquilon, le Supernas et le Caecias; autour du Solanus, le Carbas et à une certaine époque les Ornithiae; et aux extrémités de l'Eurus qui se trouve au milieu, lEuricircias et le Volturnus. Mas il y a encore en plus fleuves, des bourrasques de montagne?

VITR, 1, 6, 4-10; Nonnullis placuit esse uentos quattuor: ab oriente aequinoctiali Solanum, a meridie Austrum, ab occidente aequinoctiali Faloonium, ab septentrionali Septennicus. Sed qui a diam exemplum conlocauit Athenis turrem marmoream octagonon et in singulis lateribus octagoni singulorum uentorum imagines excalptas contra suos cuiusque flatus designauit $[\ldots]$ Itaque sunt conlocati inter Solanum et Austrum, ab oriente hiberno Eurus, inter Austrum et Fauonium, ab occidente hiberno Africus, inter Fauonium et Septentrionem, Caurus, quem plures uocant Chorum, inter Septentrionem et Solanum, Aquilo [...] Fortasse mirabuntur hi qui multa uentorum nomina nouerunt quod a nobis expositi sunt

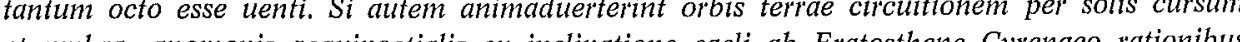

Manilius (4, 589-594)

Manilius esquisse seulement une rose des vents au livre IV de ses Astronomica à propos de la représentation du ciel divisé en quatre parties:

Autant de vents se précipitent des mêmes régions et se livrent entre eux de vains combats. Le rude Boreas s'élance du pôle 10, l'Eurus s'entuit à son coucher. Entre eux à partir de chaque région intermédiaire, deux brises expirent des souffles similaires avec d'autres noms"

Le poète ne donne pas les noms des vents intermédiaires et l'expression par laquelle il les désigne est obscure: hos inter binae mediis e partibus aurae. Manilius veut-il dire qu'il y a deux vents soufflant de chacune des parties comprise entre deux vents cardinaux (on aurait alors un système à douze vents), ou veut-il dire qu'il y a deux couples de

mathematicis et geometricis methodis esse inuentam ducentorum quinquaginta duum milium stadium, quae fiunt passus trecenties et decies quinquies centena milia, huius autem octaua pars quam uentus tenere uidetur est triciens nongenta triginta septem milia et passus quingenti, non debebunt mirari si in tam magno spatio unus uentus uagando inclinationibus et
recessionibus uarietates mutatione flatus faciat. Itaque dextra et sinistra Austrum, Leuconotus et Altanus flare solet; Africum, Libonotus et Subuesperus; circa Fauonium, Argestes et certis temporibus Etesiae; ad latera Cauri, Circias et Chorus; circa Septentrionem, Thracias et Gallicus; dextra ac sints ta Aquilonem, Supernas et Caeclas; circa Solanum, Carbas et cens. Sunt autem et alia plura nomina flatusque uentorum e locis aut fluminibus as el Volturprocellis tracta. Nous avons commenté cette rose dans notre édition du Live I de Vitruve pour la Collection des Universités de France (Paris, Les Belles Lettres, 1990).

sffit à désigner le pôle nord; cf. Lucr. 6,720.

11 MANIL. 4, 589-594: ... Totidem uenti de partibus isdem erumpunt secumque gerunt per inania bella Asper ab axe ruit Boreas, fugit Eurus ab ortu,
Auster amat medium solem Zephyrusque profectum. Hos inter binae mediis e partibus aurae

Rapprocher ce passage d'Oxide (Met. 1,61-66) qui utilise exactement les mêmes dénominations: Eurus ad Auroram Nabataeaque regna recessit Pesper et occiduo quae litora sole tepescunt Xima sunt Zephyris Scythiam septemque trion horrifer inuasit Boreas. Contraria tellus. nubibus assiduis pluuioque madescit ab Austro.

Sénèque connait les vers d'Ovide et les cite en Nat. 5, 16,1. 


\section{PH. FLEURY}

vents soufflant des régions intermédiaires (système à huit vents)? Sénique qui utilise aussi la notion de bini uenti dependant des vents

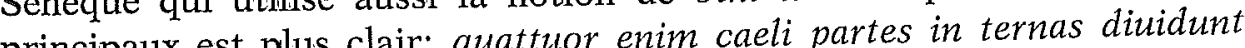
principaux est plus clains nettethingulis wentis binos subpre varronienne ment vers un systene a douze vents selo in (cf. infra). Cetlo notion de acouple de ventss ents: les réapparaît plusieurs fois dans les descriptions de roses auteurs partent des quatre vents cardinaux et obtiennent ainsi une rose à douze vents. Vitruve est le seul auteur connu qui parte dune rose à huit vents en y appliquant ce systeme pour obtenir donc une rose à vingt-quatre vents. Quel que soit le nombre total des vents sur la rose de Manilius, son ébauche est interessante comparee à celle de Vitruve dont elle est assez proche dans le temps ${ }^{12}$, car, pour trois des quatre vents cardinaux, il utilise les noms grecs: Boreas, Eurus et Zephyrus, là où Vitruve utilise des dénominations latines: Septentrio, Solanus et Fauonius ${ }^{13}$.

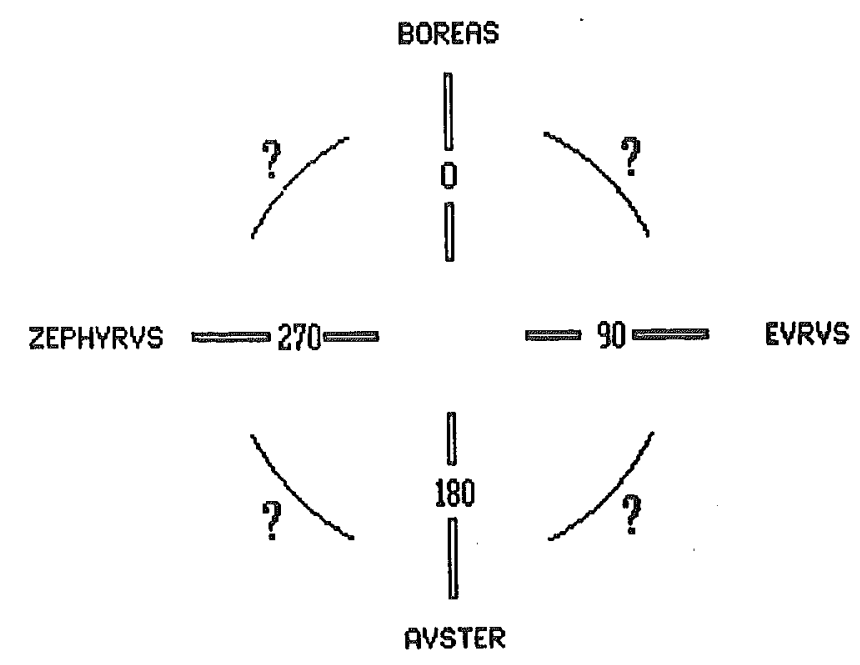

Fig. 2. La rose de Manilius (Irr siècle p.C.)

12 Les livres I et II des Astronomica ont été écrits sous Auguste, mais après 9 p.C. Le livre IV a été écrit sous Tibère: cf. M. Manilii Astronomica, ed. G. P. GooLd, Leipzig, Teubner, 1985.

${ }_{13}$ Vitruve n'utilise jamais les dénominations Boreas et Zephyrus; quant à l'Eurus, il en fait un vent de SE.
Sénèque $($ Nat. $5,16,1-17,5)$

Le Livre $\mathrm{V}$ des Questions Naturelles de Sénèque est entièrement consacré aux vents; certains manuscrits l'intitulent d'ailleurs De Ventis ou De Vento. Le philosophe examine d'abord plusieurs définitions du vent, ses causes, puis, avant de terminer par des considérations morales sur l'utilisation des vents par l'homme, il décrit une rose à douze vents en donnant à chaque vent cardinal deux vents collatéraux selon une en donnant à chaque vent cardinall deux
méthode déjà appliquée, dit-il, par Varron:

Les vents sont quatre, partagés entre l'est, l'ouest, le midi et le nord; les autres, que nous appelons de différents noms, sont attachés à ceux-ci [...] Certains donnent douze vents. Ils divisent en effet chacune des quatre régions du ciel en trois et adjoignent deux vents subalternes Varron, un homme consciencieux, et non sans raison [.] Celui qui vient du levant équinoxial est appelé chez nous Subsolanus, les Grecs l'appellent Apheliotes. Du levant d'hiver vient l'Eurus que ceux de chez nous ont appelé Vulturne - Tite-Live lui donne ce nom à propos de la bataille peu favorable aux Romains dans laquelle Hannibal vainquit, avec l'aide du vent et de l'éclat du soleil qui éblouissait les yeux de ses ennemis, notre armée placée face au soleil qui se levait et face au vent. de cité et ne donne plus l'impression d'un intrus dans notre langue. Du levant solsticial nous arrive un vent que les Grecs appellent Caecias et qui n’a pas de nom chez nous. L'occident équinoxial nous envoie le Favonius, que t'appelleront Zéphyr même ceux qui ne savent pas le grec. Du couchant solsticial vient le Corus que certains appellent Argestes - ce n'est pas mon avis: le Corus est violent et se dechaine toujours dans le même sens; l'Argestes est ordinairement doux et sert aux voiliers qui s'en vont, comme à ceux qui reviennent-, du couchant Libs. Du côté nord l'Aquilon est le vent supérieur i4. le Septentrion est le vent médian: le Thracias 15 est le vent inférieur; il lui manque un nom chez nous. Du côté de l'axe austral il y a l'Euronotus $^{16}$, puis le

${ }_{14}$ Sénèque pense à la représentation graphique de la rose des vents avec l'est en haut: du côté gauche du dessin le nord occupe le milieu, le nord-est est au-dessus, le nord-ouest au-dessous

15 Sur les graphies Thracias ou Thrascias, cf. PH. Freury, Vitruve, Livre I (op. cit.), p. 180 n. 10.

16 Certains manuscrits donnent Austronotus, un étonnant pléonasme. 
PH. FLEURY

Notus, Auster en latin; ensuite, le Leuconotus qui n'a pas de nom chez nous 17 . Nous admettons ce chiffre de douze vents, non qu'il y en ait partout ce nombre, - en effet lorientavantage. [...] Certains vents son mais parce qu'il n'y en a nulle

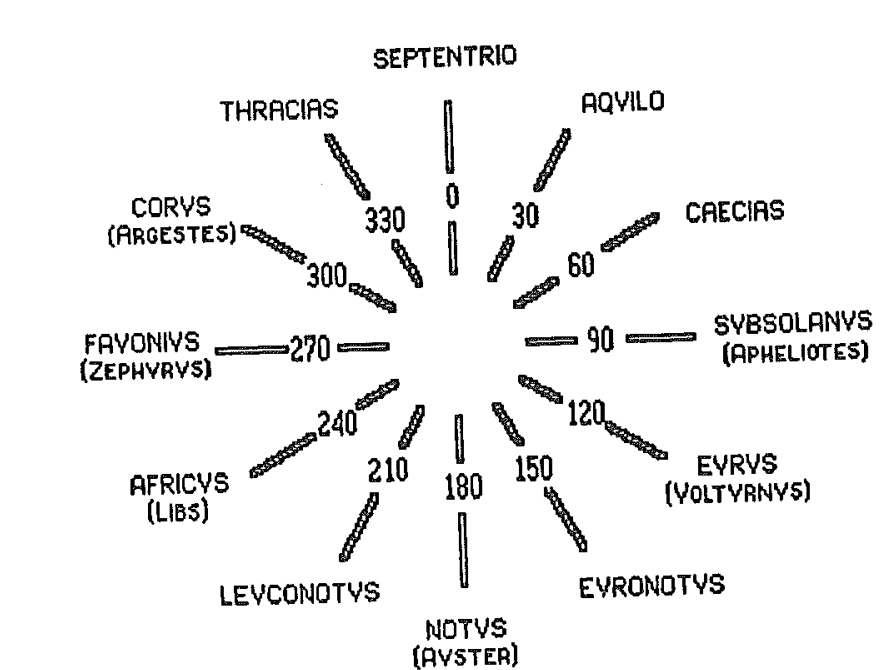

3. La rose à douze vents de Sénèque (I ${ }^{\text {er }}$ siècle $p . C_{\text {. }}$ ). (= Varron, Ir siècle $a . C$.

17 Végèce donne Albus Notus, cf. infra.

18 SEN., Nat. 5, 16, 1-17, 5: Venti quattuor suint, in ortum, occasum, meris trionemque diuisi; cetert, quos un duodecim faciunt. Quc are Varro, uir diligens, illos ordinat, nec sine causa [...] Qui surgit subpraefectos dani. Criali Subsolanus apud nos dicitur, Graeci illum Aphelioten uocant. Ab oriente hiberno Eurus exit quem nostri uocauere Vulumum - a Lims holem orientem appellat in illa pugna Romanis parum prospera in qua Hannibal an fulgoris praestringentis exercitum nostrum et contra uentum conshinum ann - sed et Eurus iam ciuitate donafus oculos hostium uicit; Varro quoque hoc est et nostro sermoni non tamquam alline est. Aequinoctialis occidens Fauonium mittit, quem Graeci appellant; apud nob etiam qui graece nesciunt loqui. A solstitiali occidente Coru Zephyrum esse dicendam Argestes dicittur, - mihi non videtur, quia Cort uiolenta uis est et in

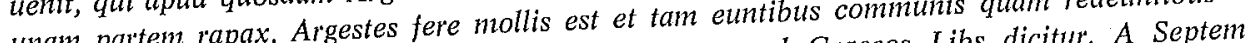
ab occidente hiberno Africus furibundus et ruens; apud Graecos Liss. huic deest apud nos trionali latere summus est Aquilo, medius Septemin

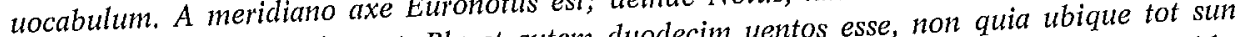
qui apud nos sine nomine est. Placet aulem derolecil - quosdam enim inclinatio toprii ...
LES ROSES DES VENTS LATINES

Pline l'Ancien (Nat. 2, 105-134)

Le livre II de l'encyclopédie de Pline l'Ancien est consacré à la cosmologie. L'exposé sur les vents occupe un peu plus du dixième de ce volume. On y retrouve des thèmes déjà présents dans le traité de Sénèque: définitions du vent, origine, rose des vents, vents locaux, et même quelques lignes morales (§ 118) sur le mauvais usage de la navigation pour l'homme. Pline donne en plus un calendrier précis pour l'apparition de chaque vent:

Les Anciens observèrent quatre vents en tout, correspondant à autant de régions du monde (aussi Homère lui-même n'en nomme-til pas davantage), système trop grossier, à ce qu'on jugea bientôt; lâge suivant en
ajouta huit, système trop subtil et fractionné; les successeurs adoptè rent un compromis, ajoutant au système bref quatre vents pris au système le plus copieux. $\mathrm{ll}$ y en a donc deux pour chacune des quatre régions du ciel: au levant equinoxial le Subsolanus, au levant d'hiver le Vulturne; les Grecs appellent le premier Apeliotes, le second Eurus; au midi lAuster et au coucher dhiver lAfricus, les Grecs les nomment Corus; les Grecs les appellent Zéphyr et Argestes; au nord, le Septentrion et entre ce dernier et le lever d'été l'Aquilon, en grec Aparctias et Boreas. Le système le plus copieux avait intercalé quatre vents entre ceux-ci: le Thracias, à mi-chemin entre le nord et le coucher d'été; de même le Caecias entre l'Aquilon et le lever équinoxial, c'est-à-dire au lever d'eté; le Phénix, entre le lever d'hiver et le midi; de même, diaire entre le midi et le, couchant d'hiver. diaire entre le midi et le couchant d'hiver. Et ce n'est pas tout: d'autres l'Eurus et le Notus, l'Euronotus. Il y a aussi des vents particuliers à certaines régions ${ }^{19}$.

19 PLINE, Nat. 2, 119-120: Veteres quattuor omnino seruauere per totidem mundi partes (ideo nec Homerus plures nominat), hebeti, ut mox indicatum est, ratione; secuta aetas octo addidit nimis subtili atque concisa; proximis inter utramque media placuit, ad brettem ex numerosa additis quattuor. Sunt ergo bini in quattuor caeli partibus: ab oriente aequinoctialt
Subsolanus, ab oriente brumali Volturnus; illum Apelioten, hunc Graeci Eurum appellant Subsolanus, ab oriente brumali Volturnus; illum Apelioten, hunc Graeci Eurum appellant;
a meridie Auster et ab occasu brumali Africus; Notum et Liba nominant; ab occasu aequia meridie Auster et ab occasu brumali Africus; Notum et Liba nominant; ab occast aequi-
noctiali Fauonius, ab occasu solstitiali Corus; Zephyrum et Argesten uocant; a septentrionibus Septentrio, interque eum et exortum solstitialem Aquilo, Aparctias et Boreas dicti. Numeriosior ratio quattuor his interiecerat: Thracian media regione inter Septentrionem et occasum solstitialem; itemque Caecian media inter Aquilonem et exortum aequinoctialem ab ortu solstitiali; Phoenica media regione inter ortum brumalem et meridiem; item inter Liba et Notum conpositum ex utroque medium inter meridiem et hibernum occidentem Libonotum. Nec finis: all quippe Mesen nomine etiamnum addidere inter Borean et Caecian, et inter Eurum Nolumque Euron nomine etlamnum addidere inter Borean et Caecian, et inter 
La description de Pline est intéressante en ce sens qu'elle présente à la fois une rose à huit vents et une rose à douze vents et que lauteur considère la plus copieuse comme antérieure à la plus simple. Comme les autres auteurs Pline part de la rose à quatre vents, mais il faut prêter une attention toute particulière à l'expression suivante, destinée à introduire la rose à huit vents: sunt ergo bini in quattuor cael partibus; nous retrouvons la notion ubinairen déjà présente explicitepartibus, ment chez Manilus ou Sén a or elle Il nest donc plus posible de pars d'uble a une rose de huit vers. partir d'une rose à quatre vents sur laquelle deux vents auxiliaires son accolés à chaque vent principal, bien quil ressonte implicitenent du texte de Pline que les choses ont du se passer comme cela chronologiquement. $D^{\prime}$ tn point de vue synchronique on ne peut rendre compte de cette notion binaire avec la rose de huit vents qu'en abandonnant l'idée de «direction de vent» pour parler de «sect était déjà présente chez Vitruve quand il décrivait la tour des Vents d'Athènes et il est bien évident que le système d'Andronicus de Cyrrho repose sur le concept de secteur et non de direction precise. Pour comprendre la phrase de Pline, il faut donc partir de l'idée d'un cercle divisé d'abord en quatre secteurs: nord, est, sud et ouest. Dans le système "simple» (hebes dit Pline) à quatre vents, le secteur nord va du nordouest au nord-est, le secteur est du nord-est au sud-est, etc. Dans le système à huit vents tel que le présente Pline tout devient complique systembè à hilis ot concisa) car non seulement les quatre régions du ciel ne (subtilis et concisa), car ques le presente Pline tout devient coms peuvent plus être orientes de facon carcinale, mas eles sont ingales. Observons en effet avec precision les groupennents binares de pline: pour le secteur $E$, il nous donne le Subsolanus et le Voltumus, un vent $d^{\prime} E$ et un vent $d^{\prime} E S E$, la région $E$ ne peut donc se situer symetriquement autour du levant équinoxial. Pour le secteur S, il nous donne l'Auste et l'Africus, un vent de $S$ et un vent d'WSW ('écart entre les bissectrice des deux secteurs est de $60^{\circ}$ ). Pour le secteur W, le Favonius et le Corus, un vent $d^{\prime} W$ et un vent $d^{\prime} W N W$. Pour le secteur $N$, le Septentrion et l'Aquilon, un vent de $\mathrm{N}$ et un vent de NNE. Le déséquilibre est flagran et il s'accorde mal avec le concept binaire qui est le point de départ de Pline et qui aurait dû le conduire à une division du cercle en parties égales. Il y a donc ici probablement superposition de deux concepts ou la trace d'une gêne de Pline (qui connaissait bien la mer et donc les noms et les vraies directions des vents) face à une rose purement nónsótrique. La logique gcoćtique du systeme l'aurait en effet con-

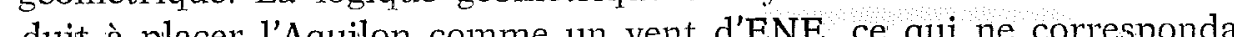
pas à la réalité.

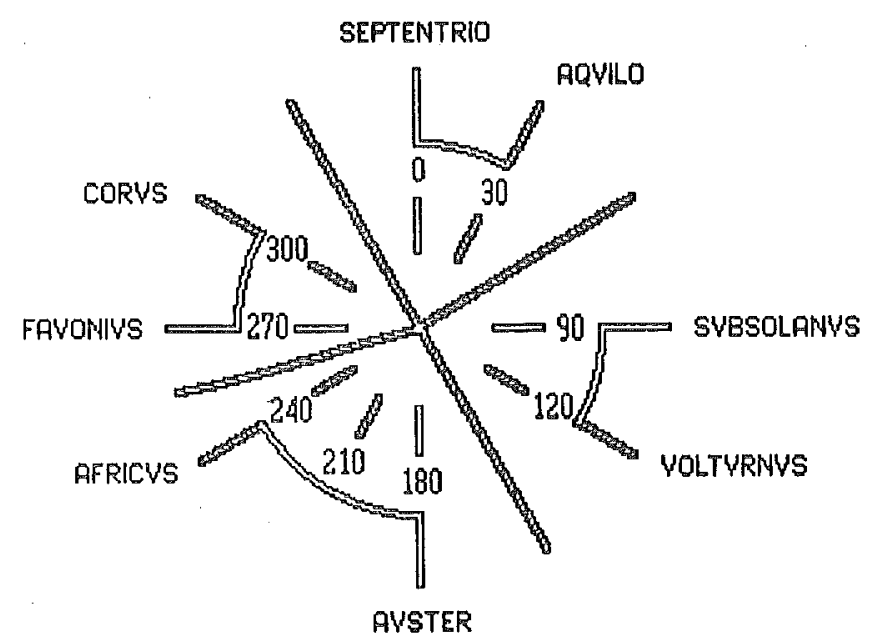

Fig. 4. Les groupements binaires de Pline sur sa rose à huit vents.

Pline aura beaucoup moins de scrupules au livre XVIII (consacré à l'agriculture) quand il s'agira de présenter une rose de huit vents l'usage du monde paysan. Là il partagera le cercle en huit parties égales et il n'hésitera pas à placer l'Aquilon à $45^{\circ}$, point qu'il appelle «le lever d'été»... Mais cette rose du livre XVIII ne doit pas être interprétée autrement que comme une simplification. Le but de Pline dans ce passage est seulement de donner des conseils aux agriculteurs sur les bienfaits et les méfaits des vents selon la direction dans laquelle ils soufflent. L'orientation précise perd sa signification ${ }^{20}$.

Une autre phrase pose problème à la fin de la description de Pline: "d'autres ont encore ajouté le Meses entre le Boreas et le Caecias et l'Euronotus entre l'Eurus et le Notus». Pline ferait-il état d'une rose à quatorze vents? La dissymétrie n'est pas gênante, nous la trouvons chez Aristote dans le sens nord-sud ${ }^{21}$. La place du Mésès à $45^{\circ}$ entre l'Aquilon et le Caecias est acceptable, peut-être même avons-nous là l'Aquilon et le Caecias est acceptable, peut-être même avons-nous
l'indice que Pline connaissait, ou avait eu entre les mains, un système de division en vingt-quatre vents avec des secteurs de $15^{\circ}$ comme chez

20 Toutefois le texte du liyre XVIII est intéressant par les épithètes que Pline donne à chaque vent et il serait utile dans une étude sur les caractérisations des vents (c. infra, n. 49).

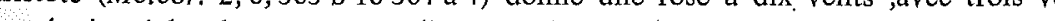


Vitruve. Mais où situer l'Euronotus? L'expression de Pline inter eurum et notum est incompréhensible, puisque nous avons déjà là le Phoenix. Faut-il comprendre inter eurum et phoenica et situer l'Euronotus à $135^{\circ}$ ? Pline se serait alors exprimé trop hâtivement. Ou faut-il voir encore ici la superposition maladroite de deux sources incompatibles entre elles?

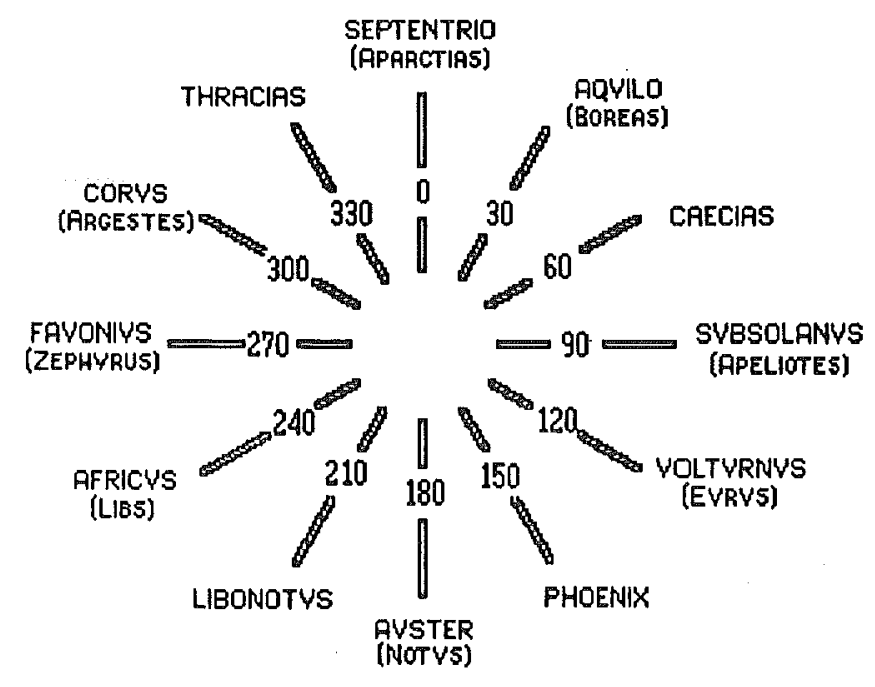

Fig. 5. La rose à douze vents de Pline $1^{\prime}$ Ancien (I'r siècle $p . C$.).

Aulu-Gelle $(2,22,3)$

Au livre II des Nuits Attiques, le chapitre XXII ${ }^{22}$ relate des propos de Favorinus sur les vents. Favorinus est un philosophe rhéteur né en Arles, peut-être sous Domitien. Il fit des tournées de conférences en Grèce et vécut à Rome sous Hadrien (qui l'exila pendant une période) et sou Antonin. Nous connaissons les titres d'une trentaine de ses cuvres (traités philosophiques, mélanges, mémoires ...). Il semble qu'il ait écri et parlé de préférence en grec. Nous ne savons si Aulu-Gelle reconstitue ci réellement de mémoire les propos de Favorinus ou si celui-ci avait

22 Le chapitre XXI est consacré à l'origine du mot septentriones; il y a donc entre

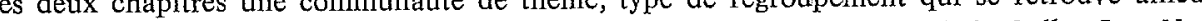
Atez Aulu-Gelle malgre Tabsence dordre general (c. R. MARACHB, Al laissé un traité sur la question. Dans les deux cas l'exposé originel peut avoir éte fait en grec; Aulu-Gelle proposerait donc ici une traduction. Le chapitre commence par une anecdote racontant qu'à la table de Favorinus il fut question un jour, lors de la lecture d'un poème, du vent nommé Iapyx. Les convives s'interrogèrent sur l'origine et la direction de ce vent et la discussion s'étendit bientôt au problème général des vents, car, nous dit Aulu-Gelle, il n'y avait pas d'accord sur leurs appellations, sur leurs auteurs et leur nombre:

Alors Favorinus parla: «Il est assez connu, dit-il, qu'il y a quatre lignes et directions dans le ciel: le levant, le couchant, le midi, le septentrion, trion se tiennent et demeurent perpétuellement au même point. En effet le soleil ne se lève pas toujours au même endroit, mais ou bien on parle de levant équinoxial quand le soleil court selon le cercle qui est appelé ionnzpovós, ou bien on parle de levant solsticial ou hivernal, ce

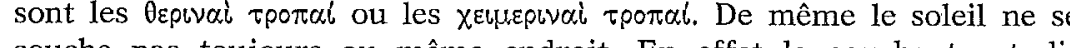
couche pas toujours au meme endroit. En effet le couchant est dit de la même manière: équinoxial, solsticial, ou hivernal. Le vent qui d'un mot formé à ce que prétendent les étymologistes, de $\dot{\partial}$ át $\tau$ T̃ noũs pécuv. Il a aussi recu des Grecs un autre nom 'Ap les marins romains, c'est le Subsolanus. Mais celui qui vient de la limite du levant au solstice d'été est dit en latin Aquilon, en grec Bopéas, et c'est pour cela, disent certains, qu'Homère l'a appele

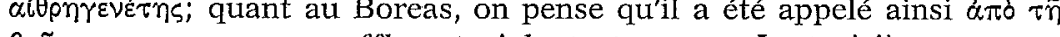
Boñs parce que son souffle est violent et sonore. Le troisième vent es celui qui souffe du levant hivernal, les Romains lappellent Volturnus, parce qu'il est situé entre l'Euris et le Notus. Ce sont donc là les tros, vents orientaux: Aquilon, Volturnus, Eurus, l'Eurus étant au milieu A l'opposé et en face de ceux-ci il y a trois autres vents occidentaux: le Chorus que les Grecs ont l'habitude d'appeler 'Apүéforns: il souffle en face de l'Aquilon; le deuxième est le Favonius qui est appelé en grec ZÉ Qupos: il souffle en face de l'Eurus; le troisième est l'Africus, $\Lambda, \psi$ en grec: il fait face au Volturnus. Ces deux regions du ciel, le levant et le couchant, opposées l'une a l'autre, ont, on le voit, six vents. Mais seul vent méridional: il est dit en latin Auster, en grec No brumeux et humide; en effet l'humidité se dit voris en grec. Le nord pour la même raison a un seul vent. Opposé à l'Auster et dirigé contre lui, il est appelé en latin Septentrionarius, en grec 'A huit vents certains en suppriment quatre, et ils disent suivre en cela l'autorité d'Homere qui ne connait que quatre vents: l'Eurus, l'Auster l'Aquilon et le Favonius, dapres les quatre parties du ciel que nous

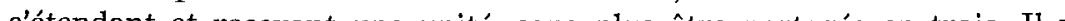




\section{PH. FLEURY}

en a d'autres qui distinguent douze vents au lieu de huit, insérant une troisième série de quatre vents au milieu des intervalles laissés autour du midi et du nord, de la même manière qu'est intercalée la deuxième Il y a en outre d'autres nes de vents, pour ainsi dire, particuliers . ${ }^{23}$.

Il est évident dans cet exposé que Favorinus ou Aulu-Gelle raisonnent à partir d'une représentation schématique de la rose des vents; ainsi aduersus ne désigne pas le vent qui souffle «en sens inverse» dan la pratique, mais le vent qui est «en face» sur le dessin (cf. fig. 6) ${ }^{24}$

23. GELL. 2, 22, 3-19: Tum Fauorinus ita fabulatus est: «Satis, inquit, notum est, limites mobilia et uaria sunt, meridies septentrionesque statu perpetuo stant et manent. Oritur enim sol non indidem semper, sed aut aequinoctialis oriens dicitur, cum in circulo currit qu

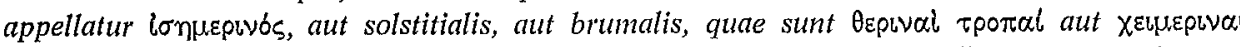
tporal. Item cadit sol non in eundem semper locum. Fit enim similter occusus eius aut aequinoctialis aut solstitialis aut brumalis. Qui tentus igitur ab oriente uerno, id est aequi-

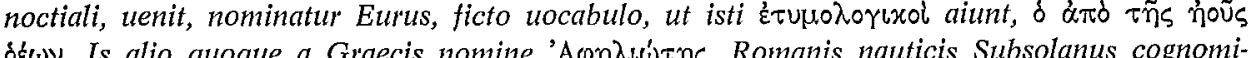

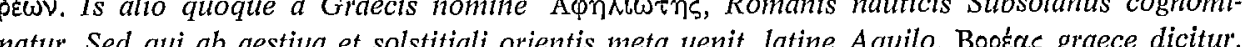

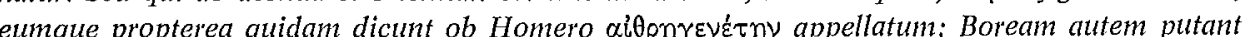
dictum $\dot{z} \pi \dot{b} \tau \tilde{n} \varsigma$ Boñs, quoniam sit uiolenti flatus et sonori. Tertius uentus, qui ab oriente hiberno spirat; Volturnum Romani uocant, eum plerique Graeci mixto nomine, quod inter Notum et Eurum sit, Eúpbvotov appellant. Hi sunt igitur tres uenti orientales: Aquilo, Volturnus, Eurus, quorum medius Eurus est. His oppositi et contrarit sunt alii tres occidui: Chorus, quem solent Graeci appellare 'ApүEбThv: is aduersus Aquilonem flat; item alter Fauonius, qui Graece Zźtevpos uocatur: is aduersus Eurum flat; Aertius Africus, qui Graece adu: aduesse sex habere uentos uidentur. Meridies autem guoniam certo atque fixo limite sest unum meridialem uentum habet: is Latine Auster, Graece Nóros nominatur, quoniam est nebulosus atque unectus; vorls enim Graece umor nominatur. Septentriones autem habent ob eandem causam unum. Is obiectus derectusque in Austrum, latine Septentrionarius, graece 'Aтxpxtlas appellatus. Ex his octo uentis alii quattuor uentos detrahunt atque id facere se dicunt Homero auctore, qui solos quattuor uentos nouerit: Eurun, Austrum, Aquilonem, Fauonium, a quattuor caeli partibus, quas quasi primas nominauimus, oriente scilicet atque occidente latioribus atque simplicibus, non tripertitis. Partim autem sunt qui pro octo

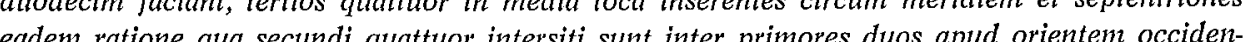
作

24 Il serait intéressant d'essayer une reconstitution des représentations antiques des roses des vents à partir des descriptions litteraires et des quelques dessins qui nous sont restés dans les manuscrits. La chose n'est pas si simple quili y parait et il semble que très rapidement les schémas de roses soient devents objets dinterêt artistique ou magique. Voir à ce sujet J. FoNTAINB, Isidore de Seville. Traite do
LES ROSES DES VENTS LATINES

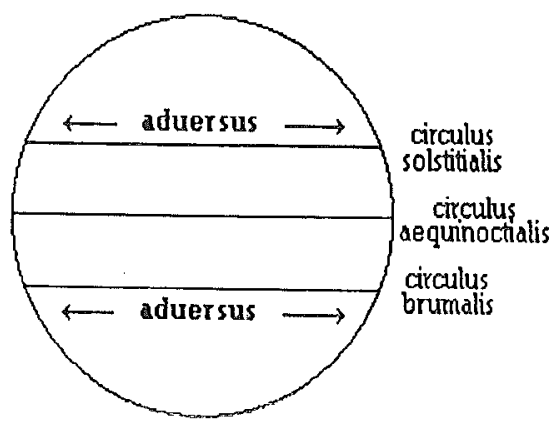

Fig. 6. Aduersus chez Aulu-Gelle.

Le mode de construction de la rose à huit vents, dans l'exposé d'Aulu-Gelle, est très clair:

1) Les quatre directions cardinales

2) Les lignes solsticiales

3) Groupements $3+3+2$

Mais quand il traduit la rose homérique: Bopéas, Zźcupos, Nótos, Eũpos ${ }^{25}$, par Aquilo, Fauonius, Auster, Eurus, il n'est plus cohérent avec son schéma, car l'Aquilo-Bopéas est devenu pour les Romains un vent de NE. La connaissance littéraire l'emporte ici sur la rigueur scientifique.

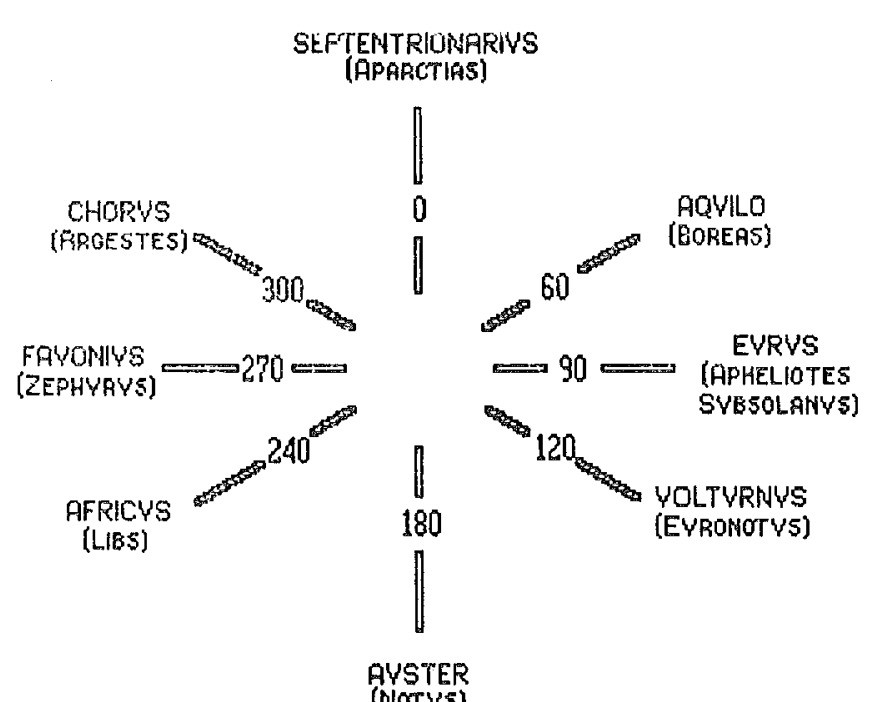

Fig. 7. La rose à huit vents d'Aulu-Gelle (II' siècle $p . C$.) (= Favorinus

25 Cf. Od. 5, 295-331 


\section{Apulée (Mund. 11, 312-315)}

Le De Mundo d'Apulée est l'adaptation latine d'un traité grec du

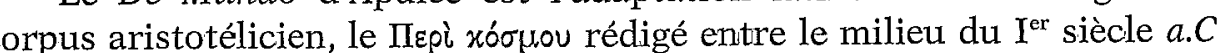
et le milieu du I Ier siècle p.C. ${ }^{26}$. Apulée a traduit presque intégralement texte grec, retranchant quelques mots ou quelques lignes parfois, modifiant ici ou là, ajoutant des remarques ailleurs. La seule addition quantitativement importante concerne précisément la théorie des vent de Favorinus, généralement considérée comme empruntée au passage d'Aulu-Gelle que nous venons de citer. Outre quelques détails de présentation, les deux textes diffèrent seulement sur la dénomination du vent du nord pour lequel Apulée ne reprend pas l'adjectif Septentrionarius utilisé par Aulu-Gelle, mais donne le nom habituel: Septentrio. Nous ne tiendrons donc pas compte de ce passage ${ }^{27}$ qu'Apulée insère dans l'adaptation du Пв the description de rose theric se coms the diverses definit a douze vents et de diune deffilignes , les vents avec asouffle alternatif, les vents dété et vents d'hiver, les ornithiae, les wents d'orage, lontagan, le tourlllon, etc. Seule nous interessera ici la description de la rose issue du Meph xophov, nous rangeons parmi les roses latines puisque l'adaptation apuleiente nous donne des traductions ou des transpositions des termes grecs:

Nous allons maintenant énumérer les noms et les directions des vents. Le levant envoie les Eurus, le nord les Boreas, le couchant les Zephyrs, nombreux. En effet, bien que l'Eurus soit le vent d'est, il reçoit cependant le nom d'Aparctias quand c'est le levant d'été qui le répand; il es appelé Apeliotes quand il est originaire du lever équinoxial; c'est l'Eurus quand il est envoyé des portes du lever d'hiver. Quant au Zéphyr, que la langue romaine connaît sous le nom de Favonius, lorsqu'il se lève de la région du coucher d'été, on l'appelle d'ordinaire Iapyx; mais celui qui est plus près de la direchion équinoxiale <est le Zéphy trion; il a pour voisin l'Aparctias; celui-ci < se dirige > vers le midi. Le Thracias et l'Argestes soufflent de la même provenance, Dans les noms des Austers, on a respecté la diversité que voici: quand les

${ }^{26}$ Cf. J. Beaujeu, Apulée. Opuscules philosophiques et fragments, texte, trad. et com., Paris, Les Belles Lettres, 1973, p. 111 et pp. IX-XXIX pour les problemes dauthenticitê du De mundo.

${ }_{28}$ = chap. XIII et XIV $(318,8-213,19)$ souffles viennent du pôle caché, c'est le Notus; l'Euronotus est celui qui se déchaine à mi-distance entre le Notus et l'Eurus; de l'autre côté

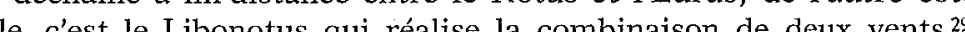

Cette rose des vents n'est que d'un intérêt médiocre en raison de problèmes de transmission du texte, problèmes de transmission d'abord du texte grec au texte latin, ensuite du texte latin jusqu'à nos jours:

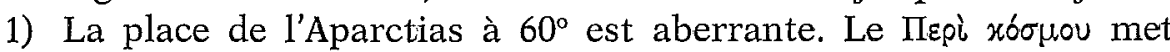
à cet endroit le Kauxias (= Caecias), mais, en le citant, Stobée commet la même erreur qu'Apulée. La faute se trouvait-elle donc dans certains

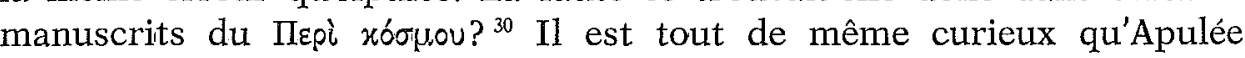
n'ait pas vu qu'il citait l'Aparctias à deux endroits différents: $0^{\circ}$ et $60^{\circ}$.

2) Deux lacunes défigurent le texte en son milieu. Le lecteur y supplée assez facilement, surtout s'il peut se reporter au texte du IIspi wófuo, mais c'est une des traces de la corruption de l'ensemble du passage.

3) Apulée écrit: Thracias et argestes sunt indidem flantes; or, dans le IIepi xóбuov, il est dit que ce sont deux vents voisins. L'auteur latin n'aurait-il pas «complètement perdu pied» comme l'affirme J. Beaujeu? ${ }^{31}$

Comme éléments intéressants de cette rose ne sont guère à retenir que le groupement $4 \times 3$ et l'emploi des pluriels: Euri, Austri, Zephyri, Boreai. Mais Apulée lui-même ne la trouvait peut-être pas satisfaisante dans l'état où il pouvait la consulter et il aurait donc ajouté pour cette raison celle de Favorinus dans la version donnée par Aulu-Gelle.

29 ApuL., Mund. 11, 312-315: Nunc nomina exsequemur regionesque uentorum. Euros oriens, Boreas septemtrio, occidens Zephyros, Austros medius dies mittit. Hos quattuor uento alii plures interflant. Nam quamuis Eurus sit uentus orientis, idem tamen Aparctias accipt procreatur: Eurus est, quando hiemalis ortus portis emittitur. Zephyrus uero, auem roman lingua Fauonium nouit, hic cum de aestiuis occiduis partibus surgit, Iapygis nomine cieri solet; at ille qui propior est aequinoctiali plagae $+\ldots$ et Aquilo, qui VII stellarum regione generatur, et huic uicinus est Aparctias; hic $+\ldots$ ad diem medium. Thracias et Argestes sunt indidem flantes. Austrorum in nominibus illa est obseruata diuersitas: namque cum de abscondito polo flatus adueniunt, Notus est, Euronotus ille qui inter Notum atque Eurum ${ }_{30}$ Cf. T. Bex ano latero Libonotus

30 Cf. J. BEAUjRU, op. cit., p. 13 


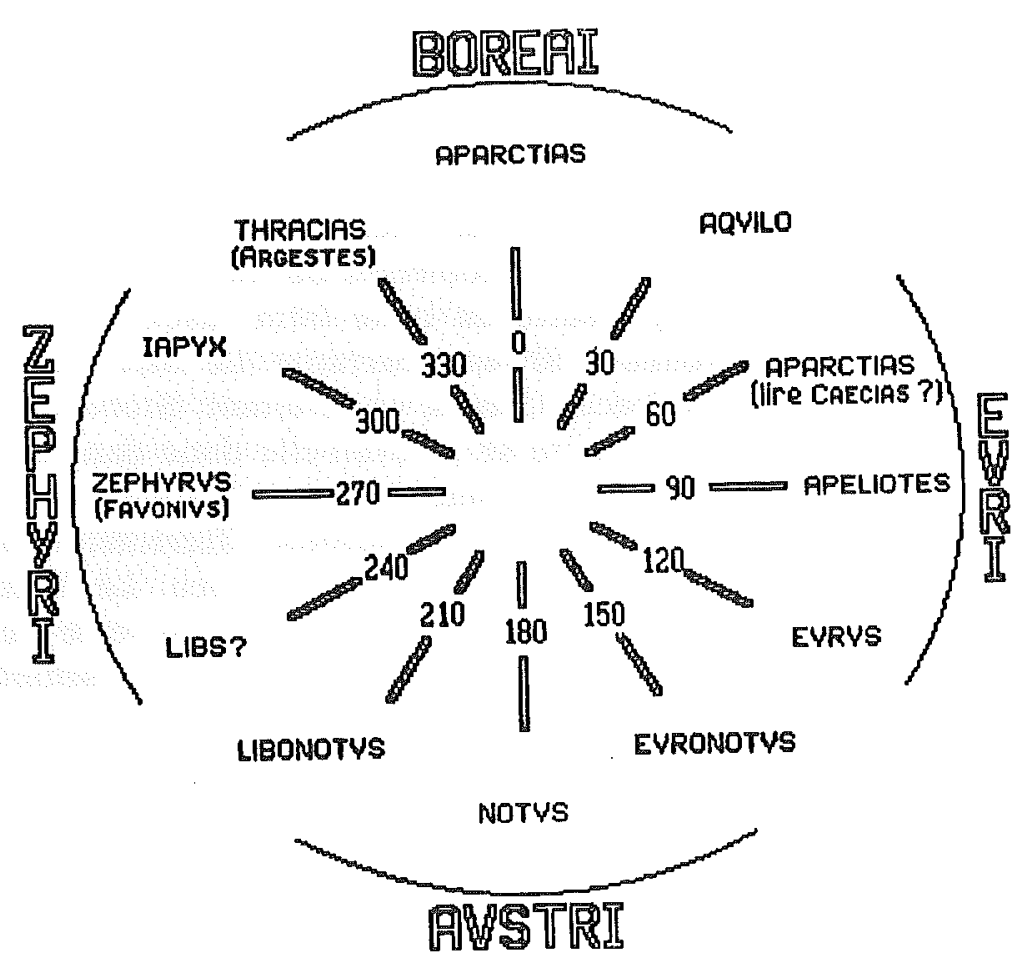

Fig. 8. La rose à douze vents d'Apulée (II' siècle p.C)

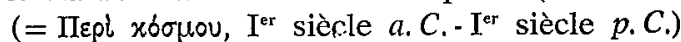

Ampélius (5)

Ampólius a dédié à Macrinus (peut-être l'empereur de 217-218 p.C.) un Liber memoralis pour qu'il sache, dit-il en introduction, quid sit mundus, quid elementa, quid orbis terrarum ferat, uel quid genus humanum peregerit. Après une définition du mundus, un exposé sur les douze signes du zodiaque et un chapitre de sideribus, Ampélius met curieusement en relation les douze vents avec les douze signes zodiacaux:

Aries in Africum, Taurus in Circium, Gemini in Aquilonem, Cancer in Septentrionem, Leo in Thrasciam, Virgo in Argesten, Libra in Zephyron

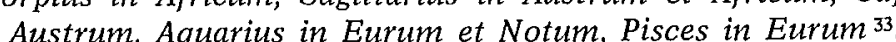

32 En tout cas le traité est postérieur à Trajan puisqu'il est question des victoires do

cet empereur sur les Daces au chap. 47.

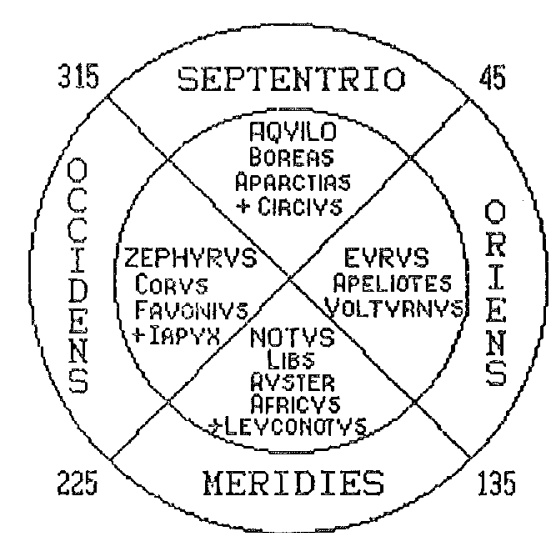

Fig. 9. Les noms de vents classés par secteurs chez Ampélius (II-III ${ }^{e}$ siècle p.C.).
Les vents naissent du mouvement de l'air et de l'sinclinaisons ${ }^{34}$ du ciel;
les vents généraux sont au nombre de quatre: l'Eurus (qui est dit aussi ${ }^{35}$ Apeliotes et Volturnus) vient du levant; du couchant vient le Zéphyr (dit aussi Corus et Favonius); l'Aquilon (dit aussi Boreas et Aparctias) vient du nord; le Notus (dit aussi Libs, Auster et Africus) vient du midi. Ce sont les quatre vents generraux; les autres sont des vents speciaux qui sont ajoutes: au Zephyr est adjoint le lapyx qui notus lorsqu'il souffle plus doucement: à l'Aquilon, le Circius leucosouffle plus violemment à travers les Gaules; de même les Étésiens qui soufflent l'été à des périodes fixes 36 .

La rose des vents d'Ampélius n'est pas reconstructible dans le détail. Il n'est pas sûr du reste qu'il ait eu une construction graphique en tête.

${ }_{34}$ Voir l'emploi d'inclinatio chez VIrr. 1,1, 10 et 1, 6, 9 .

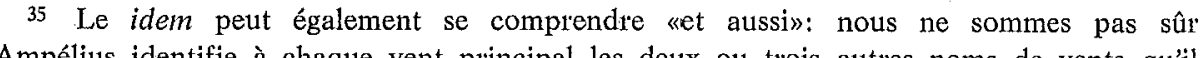
donne ensuite. Il peut aussi effectuer un regroupement des noms de vents enr quate sectert $X$ sans s'attacher à l'orientation exacte à l'intérieur des secteurs. 36 AMPEL, 5: Venti fitunt ex aeris motu et inclinatione; sunt autem generales quattuor Eurus, idem Apeliotes, idem Volturnus ab oriente; ab occidente Zephyrus, idem Corus, idem Fauonius; Aquilo, Boreas, Aparctias idem a septentrione; Notus, idem Libs et Auster a Africus a meridie. Hil sunt quathor generales; ceteri speciales adscribuntul, ut Tapyx Zephyro, loni, aum apurion 


\section{PH. FLEURY}

Les très brefs chapitres 4 et 5 n'ont apparemment d'autre intention que de donner un rapide catalogue de vents avec toutefois, dans

$$
\begin{aligned}
& \text { - définition du vent } \\
& \text { - vents généraux }
\end{aligned}
$$$$
\text { - vents particuliers. }
$$

Végèce $(4,38$ ou 5,8$)$

A la fin du livre IV, ou au début du livre $\mathrm{V}$ selon la répartition effectuée par les éditeurs, Végèce traite de la guerre sur mer et il est amené naturellement à consacrer un chapitre aux vents car, dit-ii, les liburnes ont souvent péri plus sûrement par la force des vents et des liburnes ont souvent péri plus s'art de la navigation doit donc examiner flots que par celle des ennemis. Lart de la navigation

Les Anciens croyaient que selon la position des points cardinaux il ry tes régions du ciel, mais l'époque suivante, grâce à l'expérience, en compte douze. Pour éviter toute hésitation nous donnons non seulement leurs noms grecs mais aussi leurs noms latins en faisant en sorte de désigner d'abord les vents principaux puis d'indiquer les vents qui leur son adjoints à droite et à gauche. C'est pourquoi nous commençons par le solstice 37 de printemps, c'est-d-dire par le levant cardinal doù vient

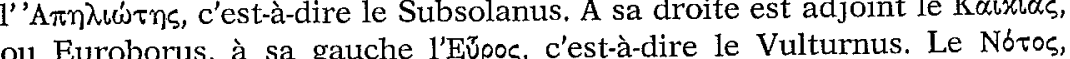
c'est-à-dire l'Auster, occupe le midi cardinal. A sa droite est adjoint le $\Lambda$ evxovóroc, c'est-à-dire le Notus blanc; à sa gauche le $\Lambda \iota \beta o v b r o s$, c'està-dire le Corus. Le Żécupos, c'est-à-dire le Subvespertinus, occupe le couchant cardinal. A sa droite est adjoint le $\Lambda$ ü, ou Africus; à sa gauche

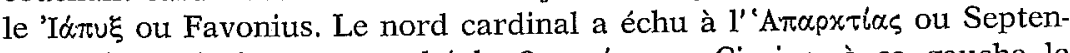
Circius, à sa gauche le Bopés $\alpha$, c'est-à-dire l'Aquilon ${ }^{38}$

37 L'expression a uerno solstitio est étrange. Ne faut-il pas lire a uerno aequinoctio, comme l'un des manuscrits, ou tout simplement $a b$ oriente uerno conme chez Aulu-Gelle?

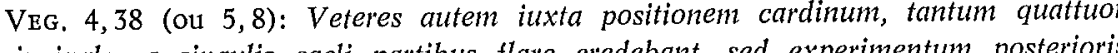
uentos principales a singens tis Horum uocabula ad summouendam dubitationem, non solum graeca, sed etiam latina protulimus, it ut, uentis principalibus declaratis, eos qui ipsis dextra laeuaque coniuncti sunt, indicemus. A uerno itaque solstitio, id est ab orientali cardine,

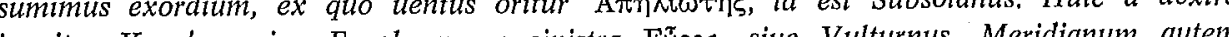

\section{LES ROSES DES VENTS LATINES}

Végèce est le seul de nos neuf auteurs à dissocier Favonius et Zéphyr. Mais s'il commence toujours par donner le nom grec, ce n'est peut-être pas seulement "pour éviter toute hésitation», mais aussi parce qu'il traduit une rose grecque. Il peut donc y avoir de l'approximation dans les transpositions.

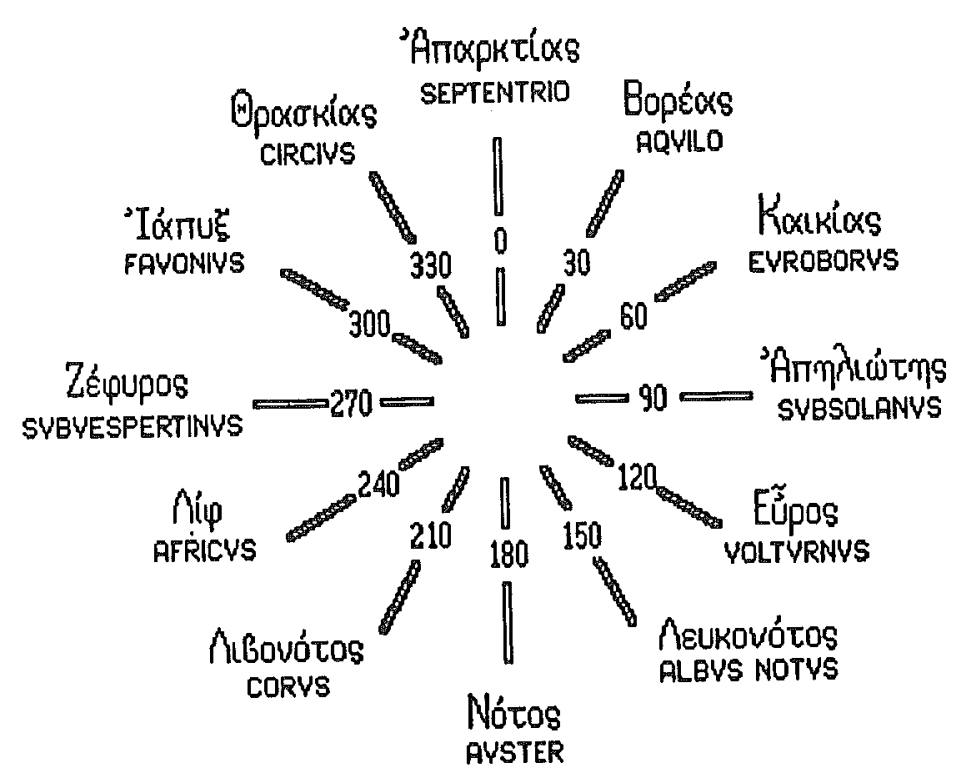

Fig. 10. La rose à douze vents de Végèce (IV॰ siècle p. $C$.

Isidore de Séville (Orig. 13, 11, 2-3; Nat. 37)

Comme Pline, Isidore de Séville donne une rose des vents à deux ndroits de son cuure: au chapitre XXXVII du De natura rerum et au livre XIII des Origines. Dans le De natura rerum elle est insérée à l'inte rieur d'une série de chapitres ${ }^{39}$ consacrés à la météorologie. Quant au livre XIII des Origines, il traite du monde et de ses parties. Le chapitre XI sur les vents est encadré par un chapitre sur l'arc-en-ciel et le

cardinem possidet Nóros, id est Auster. Huic a dextra iungitur $\Lambda$ Euxovbros, id est Albus

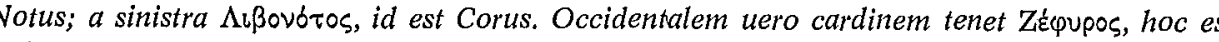

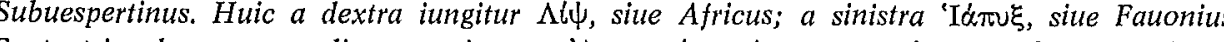

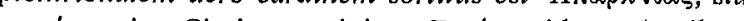

${ }_{39}$ C'es Chelus, a shistra Boplas, id est 
effets des nuages et un chapitre sur les eaux. Mais alors que chez Pline la rose du livre XVIII est d tne autre nature que celle du livre II, chez Isidore les deux roses sont semblables; seule la présentation diffère. Nous ne nous attacherons ici qu'à la première dans l'ordre chronologique, celle du De natura rerum:

e premier vent cardinal est le Septentrion, froid et neigeux: il souffe directement du pole nord et produit les froids secs et les nuages arides; if s'appelle aussi Aparctias. Le Circius, qui s'appelle aussi Thracias, grondant lui a la droite du Septention, produit les neiges ce la torstion de la gucli liać, sec et sans pluie, il ne disperse pas les nuages, mais les épaissit; par suite, ce n'est pas sans raison qu'il personnifie le diable, car il resserre les coeurs des païens par le froid de la méchancete. Le second vent cardinal est le Subsolanus appelé aussi Apeliotes; il gronde du côté du levant et est tempéré. Le Vulturnus, appelé auss Caecias, est à la droite du Subsolanus; il évapore et desseche tout. L'Eurus, qui vient du côté gauche du Subsolanus, arrose le levant de l'Auster, Auster, appele aussi Nolus, soul celnt des pluies très abondantes; c'est également lui qui épanouit les fleurs. L'Euroauster est un vent chaud qu' gronde à droite de l'Auster. L'Euronotus ${ }^{40}$ est un vent tempéré et chaud qui souffle à gauche de l'Auster. Le quatrième vent cardinal est Zéphyr, appelé aussi Favonius, qui souffle du fond de l'occident, il détend la rigueur de l'hiver par son retour délicieux et fait pousser les fleurs. LAfricus, qu'on appelle Libs, vient en grondant de la droite du Zephyr, il engendre les tempêtes et les plules, provoque les chocs des nuages, les gouste de la gauche du Favonius; quand il donne, le ciel est nuageux au levant et serein dans l'Inde. Tranquillus ${ }^{41}$ donne des appellations déterminées à certains souffles propres à une région donnée ... ${ }^{42}$.

Dans plusieurs manuscrits, le schéma représentant la rose des vents porte à cet endroit (sous une forme plus ou moins alterée) la denomination Austroafricus qui comprend mieux

Tranquillus, c'est-à-dire Suétone, est donné comme source de la notice sur les vents régionaux, mais rien ne prouve qu'il soit l'auteur de la rose tout entière comme le pense

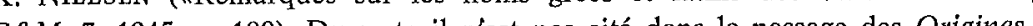
a dice et facit arida frigora et siccas nubes; hic et Aparctias. Circius, qui et Thrascias, hic a dextris Septentrionis intonans facit niues et grandinum coagulationes. Aquilo uentus. qui et Boreas uocatur, ex alto flans, gelidus atque siccus et sine pluuia, qui non discult nubes, sed stringit; unde et non inmerito diaboli formam induit, quia iniquitatis frigore gentilium corda constringit. Secundus uentorum cardinalis Subsolanus, qui et Apeliotes; hic

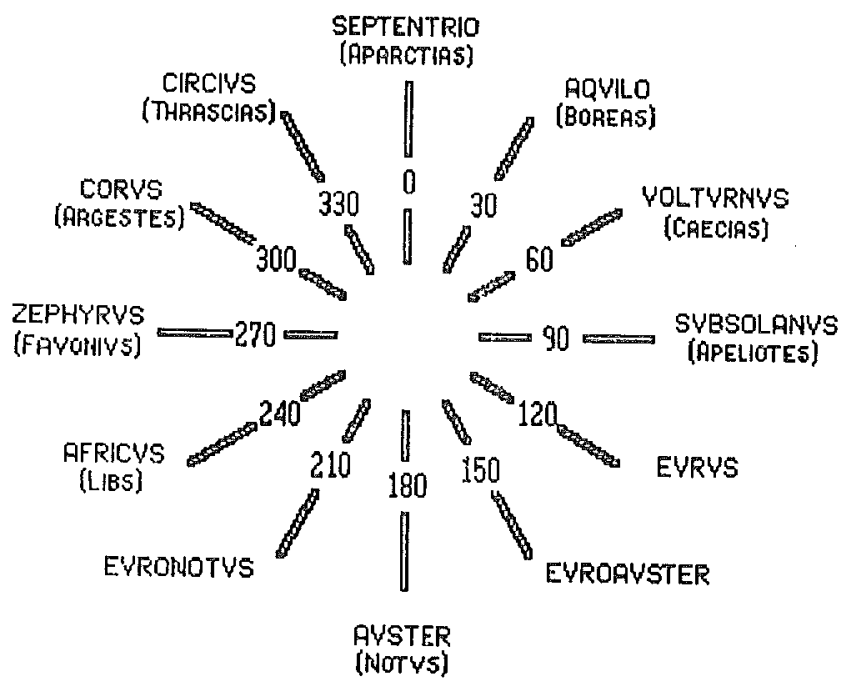

Fig. 11. La rose à douze vents d'Isidore de Séville (VII' siècle $p . C$.)

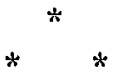

En reprenant l'ensemble de ces roses, il apparaît que l'objectif de définition et d'orientation des régions du ciel n'a pas été réalisé de façon satisfaisante à l'époque romaine. En effet plusieurs dénominations (et non des moins connues) se déplacent sur l'horizon suivant les auteurs et selon les époques. Le tableau que nous avons tracé à la fin de cet article le montre distinctement: nous avons relevé tous les noms de article le montre distinctement: nous avons releve tous les noms de
vents qui apparaissent sur les roses latines, y compris les noms grecs vents qui apparaissent sur les roses latines, y compris les noms grecs
et les variantes qui sont données par les auteurs eux-mêmes. Nous

Subsolani; hic dissoluit cuncta atque desiccat. Eurus, ex sinistro latere ueniens Subsolani, orientem nubibus inrigat. Tertius uentorum Auster, plagae meridianae cardinalis, qui et laetissimas, soluens etiam flores. Euroauster, calidus uentus, a dextris intonat Austri. Euronotus uentus temperatus, calidus, a sinistris Austri aspirat. Quartus cardinalis Zephyrus, qui et Fauonius, ab occidente interiore flat; iste hiemis rigorem gratissima uice relaxat, flores producit. Africus, qui dicitur Lips, ex Zephyri dextro latere intonans; hic generat tempestates et pluuias, et facit nubium conlisiones et sonitus tonitruorum et crebrescentium fulgorum uisus et fulminum impulsus. Corts, qui et Argestes, ex sinistra parte Fauoni adspirans; eo flatus certis appellat uocabulis 
obtenons ainsi un catalogue de quarante dénominations ${ }^{43}$ en face desquelles nous avons noté les orientations relevées chez les neuf auteurs latins ${ }^{4}$. Des variations comme celle de l'Aquilon au-dessus ou au-dessous du NE sont peu

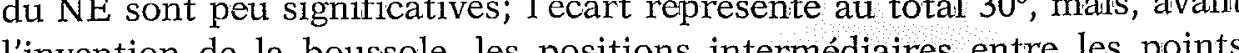
l'invention de la bousole les postions in cardinaux etaient necessairement approxination étonnantes sont les variations du Boreas qui va du $\mathrm{N}^{\circ}$ jusqu'en dessous du NE (0-60), de litur qui va de l'e au SE (90-135), ou encore celles du Leuconotus qui peut etre un vent de secteur E ou $W\left(150^{\circ}-210\right)$ ou du Volturnus qui peut etre un vent de secteur $\mathrm{N}$ ou $\mathrm{S}\left(60^{\circ}-150^{\circ}\right)$. A vrai dire ce déplacement des noms de vents sur lhorizon est normal et explicable: les textes que nous citons se datent du I ${ }^{\text {er }}$ siècle a.C. au VII siècle p.C., ils empruntent des éléments aux roses grecques antérieures et couvrent une aire géographique qui s'étend de l'Espagne aux côtes d'Asie Mineure. Comment s'étonner que, sur une telle durée et dans un tel espace, il n'y ait pas eu des évolutions linguistiques, des différences de perception locale? Le système d'orientation à partir des noms de vents ne pouvait fonctionner. Paradoxalement la rose des vents ne deviendra fiable que lorsque la procédure inverse sera suivie: lorsque les vents seront nommés à partir de l'orientation: N, NNE NE, ENE, E..

L'apparition de la boussole en Occident à la fin du XII siècle n'est L'appartio he la bou que, dans le cata teux qui reposent sur l'orientation cardinale, facile à déterminer, restent stables: Aparctias, Septentrio (-trionarius) pour le nord, Notus, Auster pour le sud, Ap(h)eliotes, (Sub)solanus pour l'est, Zephyrus pour l'ouest ${ }^{45}$. La ligne nord-sud était probablement la ligne de base et la ligne est-ouest une perpendiculaire. La ligne nord-sud est en effet facile à déterminer la nuit par l'observation de la constellation de l'Ourse (les mots Aparctia

43 L'économie du catalogue des noms de vents donné par R. BöKrR dans la Real Encyclopädie (VIII, A 2 [1958], s. $u$. "Winde», Abt. D, col. 2288-2325) nous échappe. S'il est comprehensible que soient exclus les «grands》 vents cardinaux traités ensuite dan

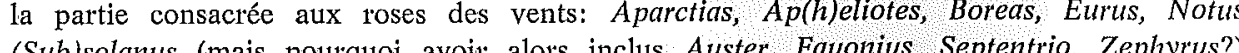
nous ne volns pourqui ont été uoubliéss: Argestes, Etesiae (Ornithiae est traité ), Euroauster, Euronotus, Gallicus, Leuconotus, Libonotus, Libs, Subuesperus (-pertinus), Supernas, Thra(s)cias.

44 Le critère de précision maximale nous est donné par la rose de Vitruve: $15^{\circ}$.

45 Si l'on accorde toute confiance au texte de Végèce, le Zéphyr est le seul nom de vent grec cardinal à ne pas avoir en de carteson et Septentrio sont un témoignage de cette pratique) et le jour par l'observation de la course apparente du soleil: Vitruve est l'auteur qui nous donne le plus clairement la méthode à utiliser avec un simple gnomon et un compas ${ }^{46}$. Pour le reste, il est facile certes de placer sur un schéma des points intermédiaires soit par division géométriqu sur un cercle, soit en seule une notion de secteur était utilisable: les quatre secteurs cardi-
naux plus quatre secteurs intermédiaires ${ }^{4}$.

L'anthologie que nous présentons peut toutefois faire l'objet d'autres lectures ${ }^{49}$. Elle est la survivance d'une littérature météorologique comme le livre $\mathrm{X}$ de Vitruve est la survivance d'une littérature mécanique. Ces littératures ont eu, nous le savons, une existence autonome dans le littératures ont eu, nous le savons, une existence autonome dans le
monde grec ${ }^{50}$. En a-t-il été de même dans le monde romain? Ou le genre monde grec ${ }^{50}$. En a-t-il été de même dans le monde romain? Ou le genre
n'a-t-il survécu qu'à l'intérieur de la littérature encyclopédique, des traités sur la nature ou des mélanges? Nous ne pouvons aborder le corpus épigraphique dans le cadre de cet article, mais, en nous limitant aux textes littéraires, il est impossible d'accepter la conclusion de K. Nielsen qui écrit: «Les auteurs latins, Vitruve, Sénèque, Pline, Suétone ${ }^{51}$, Aulu-Gelle, Végèce, malgré leurs diversités, révèlent une source commune, avant tout par leur manière d'exposer la matière, par leur essai de faire l'historique du développement, par des tours et des expressions qui passent de l'un à l'autre presque dans la même forme et surtout par la mention des vents locaux. $\mathrm{Si}$ partant de ces auteurs, on escie de reconstruire la rose des vents de Vartont, on essie de re peu pres au reculat suivant: comme source commune sinon unique des auteurs rom regarder Varron, qui dans De ora maritima a traite les vents et la rose des vents plutôt d'après des conceptions historiques et archéologiques

46 VIrR. 1, 6, 6-7.

47 Les Anciens connaissaient presque la valeur exacte de l'angle de l'écliptique à leur epoque: Vitruve par exemple $(9,7,4.5)$ donne $1 / 15^{\circ}$ de circonférence, soit $24^{\circ}$

Encore aujourd'hui les prévisions météorologiques portant sur la direction des vents n'utilisent que ces hult directions.

49 En marge des lectures scientifiques ou techniques (par exemple sur les noms d'orienseptentrio) pourrait être menée une étude des caractérisations des noms de vents. Plusies, de nos auteurs attachent à chaque vent une ou plusieurs épithètes: furibundus, callidus, humidus..., et ces caractérisations se retrouvent sur les représentations figurées, (voir par exemple la Tour des Vents à Athènes).

51 Pour K. NIELSEN, Isidore de Séville ne fait que citer Suétone (cf. supra, n. 41). 
PH. FLEURY

Tl a mentionné à la fois la rose à quatre, celle à huit, et celle à douze vents, lesquelles il a munies tant de noms recs que de noms latins. En pratique, il a suivi le système à douze vents Sa source grecque est sans doute Posidonius». Certes chez Vitruve, Sénèque, Pline et Isidore nous trouvons le plan tripartite: définition du vent, rose des vents, vents locaux, et nous trouvons une trace de ce Then Aulu-Gelle, Apulée ${ }^{52}$ et Ampellus. Certes tous les plan che l'Homère, mais ne s'agit-il pas là de ll est explicit de convention des desceit en fait qu'lles ne sont pas truction des roses des autres. Sur nos neuf aute ${ }^{53}$, toutes conpatibles seuls Séneque, Apule, Vegece et Ision ils adjoignent deux vents subaldu schema $4 \times 3$. à chaque vent car ternes et obtiennent un total des Aulu-Gelle ont des methodes tout à fait disfere vents intermédiaires quatre vents cardinaux auxquels il ajoule qu pour obtenir une rose a huit vents (celle à laquelle Puis, à partir de la rose à huit vents, 11 adopte le puint $8 \times 3$, et ajoutant à chaque vent deux vents subalternes, il obtient une rose à vingt-quatre vents. Pline part, lui, d'un principe $4 \times 2$, c'est-a-dire qu' il divise l'horizon en quatre secteurs contenant chacun delx vents. mobtien ainsi une rose à huit vents à laquelle il ajoute quatre vents pour obtenir la rose à douze vents. Mais nous avons déja montre lincoherence de la son syster elles. C'est Aulu-Gelle/Favorinus qui, scientifiquement conpatios meilleure méthode de construction et tech in $3+3+2$ : trois vents orientaux, trois vents occidentaux avec le principe $3+3+2:$ ent du sud. Mais les modes de construction de un vent du nord et un Vitruve, Pline so et Aulu-Gelle sont ine $4 \times 3$ des autres auteurs. Il ne incompatibles egalement avec le prince que nous ne

et Ampélius dont les textes ne En tealte nous devons laisser de construction de la rose.

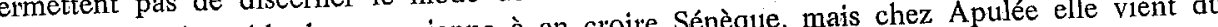

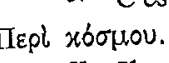

55 I1
LES ROSES DES VENTS LATINES

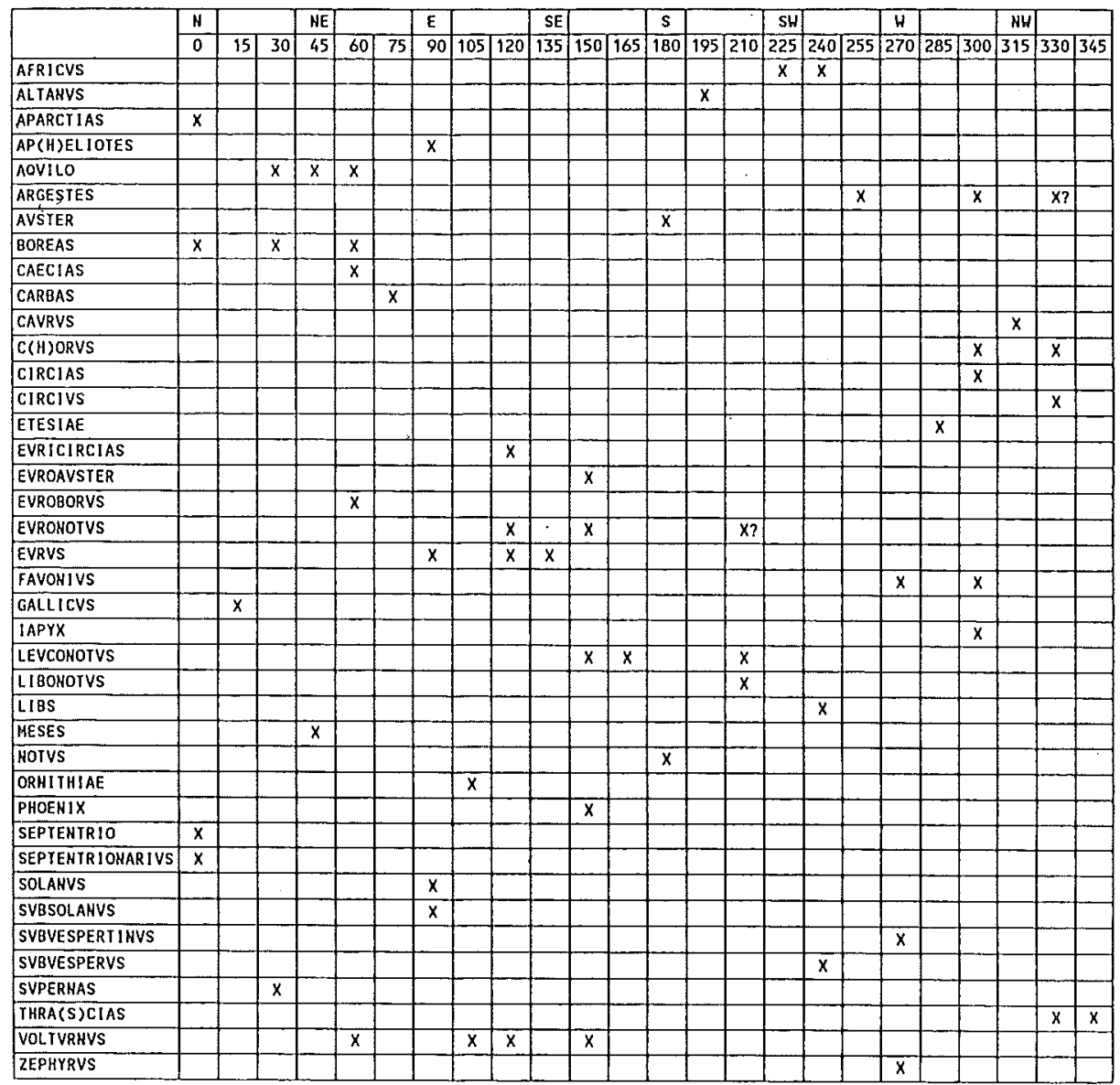

Directions des vents sur les roses latines 


\section{PH. FLEURY}

maissons pas les écrits de Varron sur les vents et encore moins ceux Posidonius qu'il fuut leur attribuer la paternité de toutes les roses vents romain tes feul à proposer roses fait ressortir loriginalite de Vitruve quans le domaine latin, mais une rose à vingt-quatre vents non seulement dans le dom également dans le domainé grec. Ses dén parfaite.

Université de Caen

SUMMARY

The object of this article is to extract in Latin literature compass cards mentioned with a scientific or technical purpose; then to present them with uniforily making a distinction between Latin and Greek tradition. We will study the writers: Vitruvitus, Manilius, Seneca, Pliny tho Elder, Aurs was of course to name each Vegetius, Isidorus Hispalensis. The wind according to its direction, butily reached in Roman times: several wind names «move» horizon. That aim was not satisfactorly reaches; variations can go from 30 to $60^{\circ}$. Those cards on the horizon interesting remnants of a meteorological literature which partly came down to us in Greek, but have almost completely disappeared in Latin. In each text there are it is impossible for all those texts to have a common source. 